\title{
Tests of the Atomki anomaly in lepton pair decays of heavy mesons
}

\author{
G. López Castro ${ }^{1, *}$ and Néstor Quintero $\odot^{2,3, \dagger}$ \\ ${ }^{1}$ Departamento de Física, Centro de Investigación y de Estudios Avanzados, \\ Apartado Postal 14-740, 07000 México D.F., México \\ ${ }^{2}$ Facultad de Ciencias Básicas, Universidad Santiago de Cali, Campus Pampalinda, \\ Calle 5 No. 62-00, Código Postal 76001, Santiago de Cali, Colombia \\ ${ }^{3}$ Departamento de Física, Universidad del Tolima, Código Postal 730006299, Ibagué, Colombia
}

(Received 14 January 2021; accepted 3 May 2021; published 27 May 2021)

\begin{abstract}
The anomalies recently reported in lepton pair transitions of ${ }^{8} \mathrm{Be}^{*}$ and ${ }^{4} \mathrm{He}$ nuclei may be attributed to the existence of a feebly interacting light vector boson $X 17$. We study the effects of this hypothetic particle in the semileptonic $H^{*} \rightarrow H e^{+} e^{-}$decays ( $H$ a $Q \bar{q}$ meson) in the framework of the HQET + VMD model. Using current bounds and the universality assumption of the $X 17$ boson to quarks, we find that decays of $D^{*+}$ and $D_{s}^{*+}$ mesons can be importantly enhanced relative to the dominant photon-mediated contributions. Dedicated experimental searches at current heavy meson factories may confirm the existence of this light boson or set stronger bounds of their couplings to ordinary matter.
\end{abstract}

DOI: 10.1103/PhysRevD.103.093002

\section{INTRODUCTION}

The existence of a light vector boson weakly coupled to Standard Model (SM) fermions has been suggested as a solution to the observed discrepancy between the SM prediction and the experimental measurement of the muon $g-2$ magnetic moment anomaly (see for example [1,2]). It may be also a good candidate as a mediator of dark and ordinary matter interactions [1,2]. Several strategies aiming their detection in different collider and fixed target experiments have not found any signal so far [3,4], but have excluded different regions in the mass and coupling strengths of parameter space. Theoretically, different models can accommodate a light vector boson and its required interactions through dimension-four kinetic mixing with SM neutral gauge bosons and their interactions with fermionic currents of SM or dark matter particles [1-3].

The anomalies recently reported in the invariant-mass spectrum and angular distribution of lepton pairs produced in ${ }^{8} \mathrm{Be}^{*}$ transitions to its ground state [5] reinforces the interest in searches of light vector bosons. The observed anomalies seems to require the existence of a spin-1 boson named $X 17$ [5-7] with mass $m_{X}=(16.7 \pm 0.35 \pm 0.50) \mathrm{MeV}$ and a relative ratio $B\left({ }^{8} \mathrm{Be}^{*} \rightarrow{ }^{8} \mathrm{Be} X\right) / B\left({ }^{8} \mathrm{Be}^{*} \rightarrow{ }^{8} \mathrm{Be} \gamma\right)=5.8 \times 10^{-6}$

\footnotetext{
glopez@fis.cinvestav.mx

nestor.quintero01@usc.edu.co
}

Published by the American Physical Society under the terms of the Creative Commons Attribution 4.0 International license. Further distribution of this work must maintain attribution to the author(s) and the published article's title, journal citation, and DOI. Funded by SCOAP ${ }^{3}$.
[7]. Couplings to standard model first-generation fermions of $\mathcal{O}\left(10^{-3}\right)$ (in units of the electron charge) required to explain this ratio is not discarded by other data. More recently, the same group seems to confirm the $X 17$ particle in studies of the $0^{-} \rightarrow 0^{+}$transitions of ${ }^{4} \mathrm{He}$ [8]. Several new physics extensions of the SM have been proposed in the literature with the required couplings to interpret the Atomki anomaly, including enlarged Higgs and/or gauge sectors (see, for instance, Refs. [7,9-13]). Despite the excitement generated by these anomalies, one must be warned that the addition of radiative corrections to the leading one photon exchange amplitude may be responsible [14] for generating the bumps reported in the angle and mass spectrum of electron-positron pairs in ${ }^{8} \mathrm{Be}^{*}$ transitions.

The almost isosinglet nature and the small mass difference of nuclei involved in ${ }^{8} \mathrm{Be}^{*}$ decay provides an ideal place to observe this light boson, in case it exists. Mixing of nuclear isospin states $[7,8,15]$ and other nuclear interference effects [16] can only partially explain the observed anomaly. Further studies in analogous systems will be very important in order to establish or discard this light boson. In the present paper, we propose the study of $H^{*} \rightarrow \mathrm{He}^{+} e^{-}$ decays, where $H\left(H^{*}\right)$ is a heavy $Q \bar{q}$ spin- 0 (spin-1) meson. Previous related studies include (i) $J / \psi \rightarrow \eta_{c} X$ decays and associated production of $J / \psi$ mesons at BESIII and Belle II experiments, recently reported in [17] and, (ii) a search proposal at $\mathrm{LHCb}$ of $D^{* 0} \rightarrow D^{0} A^{\prime} \rightarrow D^{0} e^{+} e^{-}$with displaced vertex or resonant production of the dark photon $A^{\prime}$ detailed in Ref. [18]. $H^{*} \rightarrow H e^{+} e^{-}$decays seem to be interesting to further test the Atomki anomaly: on the one hand, the mass splitting in heavy mesons is large enough (see Table I) to produce the $X 17$ boson on shell; on the 
TABLE I. Mass splittings of heavy mesons and electromagnetic couplings of $H^{*} \rightarrow H \gamma$ transitions in the HQET + VMD model. Within square brackets we show experimental values when available.

\begin{tabular}{|c|c|c|c|c|}
\hline Transition & $m_{H^{*}}-m_{H}(\mathrm{MeV})$ & $e_{Q} / m_{H^{*}}\left[\mathrm{GeV}^{-1}\right]$ & $e_{q} / m_{q}(0)\left[\mathrm{GeV}^{-1}\right]$ & $F_{H^{*} H \gamma}(0)\left[\mathrm{GeV}^{-1}\right]$ \\
\hline$\overline{D^{*+} \rightarrow D^{+} \gamma}$ & $140.603(15)$ & 0.33 & -0.85 & $-0.54 \pm 0.05[-0.47 \pm 0.06[19]]$ \\
\hline$D^{* 0} \rightarrow D^{0} \gamma$ & $142.014(30)$ & 0.33 & 1.70 & $2.11 \pm 0.10[<10.8[19]]$ \\
\hline$D_{s}^{*+} \rightarrow D_{s}^{+} \gamma$ & $143.8(4)$ & 0.32 & -0.48 & $(-0.17 \pm 0.03)[>-16.4[19]]$ \\
\hline$B^{*+} \rightarrow B^{+} \gamma$ & $45.37(21)$ & -0.063 & 1.70 & $1.64 \pm 0.09$ \\
\hline$B^{* 0} \rightarrow B^{0} \gamma$ & $45.37(21)$ & -0.063 & -0.85 & $-0.92 \pm 0.12$ \\
\hline$B_{s}^{* 0} \rightarrow B_{s}^{0} \gamma$ & $48.6\left({ }_{-1.5}^{+1.8}\right)$ & -0.062 & -0.48 & $(-0.42 \pm 0.02)$ \\
\hline
\end{tabular}

other hand, strong decays of $H^{*}$ are either very suppressed of forbidden by kinematics, leaving electromagnetic decays as dominant. Furthermore, the large amount of data produced at heavy meson factories would allow one to test the proposed channels in the near future.

The Lagrangian describing the interaction of quark and lepton flavors $f$ with the photon and the $X$ boson is $\mathcal{L}_{(\gamma, X) f f}=-e \sum_{f}\left(e_{f} A_{\mu}+\varepsilon_{f} X_{\mu}\right) \bar{f} \gamma^{\mu} f$, with couplings strengths $e_{f}$ and $\varepsilon_{f}$ given in units of the electron charge $e$. The photon and $X$ boson couplings to hadrons are described each by a single vector form factor which takes into account their structure in the momentum transfer region $4 m_{e}^{2} \leq q^{2} \leq\left(m_{H^{*}}-m_{H}\right)^{2}$, with $q=p_{e^{+}}+p_{e^{-}}$. The form factors describing the couplings of the off-shell vector particles $(V=\gamma, X)$ in $H^{*}\left(p_{H^{*}}, \epsilon_{H^{*}}\right) \rightarrow H\left(p_{H}\right) V(q)$ are defined from the hadronic amplitude

$$
\mathcal{M}_{\mu}=i e F_{H^{*} H V}\left(q^{2}\right) \epsilon_{\mu \nu \alpha \beta} \epsilon_{H *}^{\nu} p_{H}^{\alpha} p_{H *}^{\beta} .
$$

For on-shell vector particles $V$, this Lorentz-vector amplitude must be contracted with its vector polarization $\epsilon_{V}^{\mu}(q)$. The case of lepton pair production is discussed in Sec. III.

\section{II. $H^{*} H$-VECTOR VERTICES}

The form factors $F_{H^{*} H V}(q)$ are evaluated in the framework of the heavy quark effective theory supplemented with a vector meson dominance model (HQET + VDM) [20,21], which has shown to give a good description of $H^{*} \rightarrow H \gamma$ decays. Since we will normalize results for our observables to this radiative decay, we use the ratio of decay rates because they are rather insensitive to the specific $q^{2}$ dependency of the form factor. This is due to the smallness of the $H^{*}-H$ mass splitting (see Table I) compared to typical hadronic scales $\left(\sim 1 \mathrm{GeV}^{2}\right)$. Also, since the contributions of heavy quarks are $1 / m_{Q}$ suppressed, we expect that such ratios are relatively independent of constants involved in light-quark contributions through the vector meson dominance model.

For self-containedness purposes, we reproduce here the term of the Lagrangian density relevant for our calculations and definitions of couplings constants [20,21]. The strong interaction of heavy mesons are described by

$$
\mathcal{L}_{2}\left(H^{*} H V\right)=i \lambda\left\langle\mathcal{H}_{b} \sigma^{\mu \nu} F_{\mu \nu}(\rho)_{b a} \overline{\mathcal{H}}_{a}\right\rangle
$$

where $\langle\cdots\rangle$ denotes the trace in flavor space, $F_{\mu \nu}(\rho)=$ $\partial_{\mu} \rho_{\nu}-\partial_{\nu} \rho_{\mu}+\left[\rho_{\mu}, \rho_{\nu}\right]$ is the field strength tensor and $\rho^{\mu}=$ $i g_{V} \hat{\rho}^{\mu} / \sqrt{2}$ where $\hat{\rho}^{\mu}$ is the $3 \times 3$ matrix of the nonet of light vector mesons. The heavy meson field $\mathcal{H}$ is defined in terms of the pseudoscalar $\left(P_{a}\right)$ and vector $\left(P_{a \mu}^{*}\right)$ mesons fields as $\mathcal{H}_{a}=\frac{1}{2}(1+\not p)\left[P_{a \mu}^{*} \gamma^{\mu}-P_{a} \gamma_{5}\right]$, and $\overline{\mathcal{H}}_{a}=\gamma^{0} \mathcal{H}_{a}^{\dagger} \gamma^{0}$. On the other hand, the coupling of light vector mesons to the vector currents are described in terms of a single constant $f_{V}$ in the $\mathrm{SU}(3)$ flavor symmetry [20,21]:

$$
\left\langle 0\left|\bar{q} T^{i} \gamma^{\mu} q\right| V(q, \eta)\right\rangle=\eta^{\mu} f_{V} \operatorname{Tr}\left(V T^{i}\right),
$$

where $\left(T^{i}\right)_{m n}=\delta_{i m} \delta_{\text {in }}$ and $i=1,2,3$ for $q=u, d, s$ quarks, respectively. The values of coupling constant are given below.

The vector $H^{*}$ and pseudoscalar $H$ heavy mesons are composed of a $Q \bar{q}$ pair, with $Q=b, c$ and $q=u, d$, s. The hadronic matrix element of the electromagnetic current is given by [20]

$$
\begin{aligned}
& \left\langle H\left(P_{H}\right)\left|J_{\mu}^{\mathrm{em}}\right| H^{*}\left(P_{H^{*}}, \epsilon_{H^{*}}\right)\right\rangle \\
& \quad=e\left\langle H\left(P_{H}\right)\left|e_{Q} \bar{Q} \gamma_{\mu} Q+e_{q} \bar{q} \gamma_{\mu} q\right| H^{*}\left(P_{H^{*}}, \epsilon_{H^{*}}\right)\right\rangle, \\
& \quad=e\left(e_{Q} J_{\mu}^{Q}+e_{q} J_{\mu}^{q}\right),
\end{aligned}
$$

where $e_{Q}\left(e_{q}\right)$ is the electric charge of the heavy quark (light quark) in units of the positron charge, and similarly, $\left\langle H\left(P_{H}\right)\left|J_{\mu}^{\mathrm{X}}\right| H^{*}\left(P_{H^{*}}, \epsilon_{H^{*}}\right)\right\rangle=e\left(\varepsilon_{Q} J_{\mu}^{Q}+\varepsilon_{q} J_{\mu}^{q}\right)$ for the $X$ boson current.

A straightforward evaluation of the form factors in the HQET + VMD model [20] leads to

$$
\begin{aligned}
F_{H^{*} H \gamma}\left(q^{2}\right) & =\sqrt{\frac{m_{H^{*}}}{m_{H}}}\left[\frac{e_{Q}}{m_{H^{*}}}+\frac{e_{q}}{m_{q}\left(q^{2}\right)}\right], \\
F_{H^{*} H X}\left(q^{2}\right) & =\sqrt{\frac{m_{H^{*}}}{m_{H}}}\left[\frac{\varepsilon_{Q}}{m_{H^{*}}}+\frac{\varepsilon_{q}}{m_{q}\left(q^{2}\right)}\right],
\end{aligned}
$$

with the effective light "quark mass" parameter 


$$
m_{q}\left(q^{2}\right)^{-1}=-\sum_{V}\left(2 \sqrt{2} g_{V} \lambda \frac{f_{V}}{m_{V}^{2}}\right)\left(1-\frac{q^{2}}{m_{V}^{2}}\right)^{-1} .
$$

The expressions for the form factors of heavy mesons are explicitly separated in Eqs. (3) and (4) into its heavy and light quark components. In the model under consideration, the couplings of heavy quarks to the photon and $X$ boson are fixed by HQET, while the couplings to the light antiquarks are modeled by the dominance of light vector mesons [20]. For the latter, the sum extends over light vector-meson resonances $\left(V=\rho^{0}, \omega, \phi\right)$ according to the light-quark content of heavy mesons [20]. Under the assumption of the ideal mixing for vector mesons, the couplings of light $u$ and $d$ quarks are dominated by the exchange of $\rho$ and $\omega$ mesons, while the coupling of the $s$ quark corresponds to the exchange of the $\phi$ meson.

Numerical inputs for couplings constants can be found in Ref. [20] and are reproduced here for reference: $g_{V}=5.8$, $\lambda=-0.289 \pm 0.016 \mathrm{GeV}^{-1}$ (updated from new experimental inputs [19]) and $f_{V}\left(m_{V}\right)$ the decay constant (mass) of vector meson $V$. Using current experimental data for lepton-pair decays of vector mesons $V \rightarrow e^{-} e^{+}$[19], one gets $\left(f_{\rho}, f_{\omega}, f_{\phi}\right)=(0.171,0.155,0.232) \mathrm{GeV}^{2}$, with very small uncertainties. In Table I we list values for the electromagnetic form factor predicted in the HQET + VMD model at $q^{2}=0$. The quoted uncertainty is dominated by the input on the $H^{*} H V$ strong coupling $(\lambda)$ in this model (in all the predictions from this model quoted below, all the other uncertainties are very small). A comparison with the magnitude of the measured form factor (within square brackets), obtained from the measurement of the radiative decay $D^{*+} \rightarrow D^{+} \gamma$ branching fraction [19], give confidence on this model.

Let us define the following ratio of two-body decay rates:

$$
R_{X / \gamma}\left(H^{*}\right)=\frac{\Gamma\left(H^{*} \rightarrow H X\right)}{\Gamma\left(H^{*} \rightarrow H \gamma\right)}=\left|\frac{F_{H^{*} H X}\left(m_{X}^{2}\right)}{F_{H^{*} H \gamma}(0)}\right|^{2} \cdot \frac{\left|\vec{p}_{X}\right|^{3}}{\left|\vec{p}_{\gamma}\right|^{3}},
$$

where $\vec{p}_{V}$ is the momentum of the final state boson in the rest frame of $H^{*}$. This ratio exhibits two important differences with respect to the similar ratio defined in ${ }^{8} \mathrm{Be}^{*} \rightarrow{ }^{8} \mathrm{Be}$ nuclear transitions [6]. First, since $m_{H^{*}} \gg m_{q}\left(q^{2}\right)$ we have a suppression of the heavy quark relative to the light quarks contributions in Eqs. (3) and (4), which is stronger for bottom meson transition amplitudes. In order to be more explicit, and for the easy reference of the interested reader, in Table II we display the values of the two contributions that appear within square brackets in Eq. (4), by assuming $q^{2}=m_{X}^{2}$ for the square of the momentum transfer of the $X$ boson. This has the advantage that the ratio $R_{X / \gamma}\left(H^{*}\right)$ is more sensitive to the $X q \bar{q}$ couplings, which are relatively well bounded from other processes [6]. On the other hand, given the larger phase space in heavy meson decays, this ratio is not suppressed by kinematics, as it happens for decay of the ${ }^{8} \mathrm{Be}$ nucleus.

Predictions for the $H^{*} \rightarrow H X$ decay fractions require an estimate of the $\varepsilon_{Q, q}$ couplings. For the couplings of the $X 17$ boson to the quarks of the first generation we use $\varepsilon_{u} \simeq$ $\pm 3.7 \times 10^{-3}$ and $\varepsilon_{d} \simeq \mp 7.4 \times 10^{-3} \quad$ [22]. They are obtained by combining $\left|\varepsilon_{u}+\varepsilon_{d}\right| \approx 3.7 \times 10^{-3}$, obtained in Refs. [6,7] from the ${ }^{8} \mathrm{Be}^{*}$ anomaly, with the null results on searches of the $\pi^{0} \rightarrow X \gamma$ by the NA48/2 experiment [23], which translates into the constraint $\left|2 \varepsilon_{u}+\epsilon_{d}\right| \leq 8 \times$ $10^{-4}$ [22] for the $X 17$ boson couplings. By assuming the NA48/2 constraint to be exactly zero, namely the "protophobic" assumption (see however [24]), one gets the results used in this paper. On the other hand, the limits on the coupling to electrons can be obtained $0.2 \times 10^{-3} \lesssim\left|\varepsilon_{e}\right| \lesssim$ $1.4 \times 10^{-3}$ from beam dump experiments at SLAC and measurements of the $g-2$ anomalous magnetic moment of the electron according to Ref. [22]. Our study requires the knowledge of second- and third-generation couplings, namely strange $\varepsilon_{s}$, charm $\varepsilon_{c}$, and bottom $\varepsilon_{b}$. A priori these parameters are independent [7], and need not be related to the first-generation couplings. Our simplest starting assumption is universality of down- and up-type quark $\varepsilon_{f}$ couplings, thus, we will take $\varepsilon_{c}=\varepsilon_{u}$ and $\varepsilon_{b}=\varepsilon_{s}=\varepsilon_{d}$; henceforth, our results will be obtained under this assumption [6,7]. Values of the $H^{*} H X$ couplings and $R_{X / \gamma}\left(H^{*}\right)$ ratios for these transitions are given in Table II. The ratios are larger than the ones in the nuclear case mainly due to the unsuppressed phase space for $X 17$ production.

TABLE II. The $H^{*} H X$ form factors evaluated at $q^{2}=m_{X}^{2}$ and ratio $R_{X / \gamma}$ defined in Eq. (6).

\begin{tabular}{lccrr}
\hline \hline Transition & $\varepsilon_{Q} / m_{H^{*}}\left[\mathrm{GeV}^{-1}\right]$ & $\varepsilon_{q} / m_{q}\left(m_{X}^{2}\right)\left[\mathrm{GeV}^{-1}\right]$ & \multicolumn{1}{c}{$F_{H^{*} H X}\left(m_{X}^{2}\right)\left[\mathrm{GeV}^{-1}\right]$} & $R_{X / \gamma}\left(H^{*}\right)$ \\
\hline$D^{*+} \rightarrow D^{+} X$ & $1.84 \times 10^{-3}$ & $-1.89 \times 10^{-2}$ & $(-1.76 \pm 0.11) \times 10^{-2}$ & $1.1 \times 10^{-3}$ \\
$D^{* 0} \rightarrow D^{0} X$ & $1.84 \times 10^{-3}$ & $9.43 \times 10^{-3}$ & $(1.17 \pm 0.05) \times 10^{-2}$ & $3.0 \times 10^{-5}$ \\
$D_{s}^{*+} \rightarrow D_{s}^{+} X$ & $1.75 \times 10^{-3}$ & $-7.83 \times 10^{-3}$ & $(-0.91 \pm 0.06) \times 10^{-2}$ & $3.1 \times 10^{-3}$ \\
$B^{*+} \rightarrow B^{+} X$ & $-1.39 \times 10^{-3}$ & $9.43 \times 10^{-3}$ & $(0.81 \pm 0.05) \times 10^{-2}$ & $1.9 \times 10^{-5}$ \\
$B^{* 0} \rightarrow B^{0} X$ & $-1.39 \times 10^{-3}$ & $-1.88 \times 10^{-2}$ & $(-2.03 \pm 0.10) \times 10^{-2}$ & $4.0 \times 10^{-4}$ \\
$B_{s}^{* 0} \rightarrow B_{s}^{0} X$ & $-1.37 \times 10^{-3}$ & $-7.83 \times 10^{-3}$ & $(-0.92 \pm 0.04) \times 10^{-2}$ & $4.1 \times 10^{-4}$ \\
\hline \hline
\end{tabular}




\section{LEPTON PAIR PRODUCTION}

The decay amplitude for lepton pair production $H^{*}\left(P_{H^{*}}\right) \rightarrow H\left(P_{H}\right) e^{+}\left(p_{+}\right) e^{-}\left(p_{-}\right)$is the coherent sum of the photon and $\mathrm{X}$-boson mediated amplitudes $\mathcal{M}\left(H^{*} \rightarrow H e^{+} e^{-}\right)=\mathcal{M}_{\gamma}+\mathcal{M}_{X}$, where $(V=\gamma, X)$ :

$$
\mathcal{M}_{V}=-e^{2} G_{H^{*} H V}\left(q^{2}\right) \epsilon_{\mu \nu \alpha \delta} \ell^{\mu} \epsilon_{H^{*}}^{\nu} P_{H}^{\alpha} P_{H^{*}}^{\delta},
$$

where $\ell_{\mu}=\bar{u}\left(p_{-}\right) \gamma_{\mu} v\left(p_{+}\right)$is the leptonic current and $G_{H^{*} H \gamma}\left(q^{2}\right)=-F_{H^{*} H \gamma}\left(q^{2}\right) / q^{2}, G_{H^{*} H X}\left(q^{2}\right)=\varepsilon_{e} F_{H^{*} H X}\left(q^{2}\right) /$ $\left(q^{2}-m_{X}^{2}+i m_{X} \Gamma_{X}\right)$. In numerical evaluations throughout this paper we use $\alpha_{\mathrm{em}}=\alpha(0)$, the fine-structure constant, because according to Table I the maximum value of the squared photon momentum is not large $\left[q_{\max }^{2}=\right.$ $\left.\left(m_{H^{*}}-m_{H}\right)^{2}\right]$. On the other hand, running effects between $q^{2}=0$ and $q_{\max }^{2}$ are very small compared with the present and forthcoming experimental accuracies which, in the absence of real estimates, we will assume to be not better than $5 \%$ for the branching fractions.

As in Refs. [6,7], we assume negligible decays of the $X 17$ boson into neutrino channels, such that its full width is given by

$$
\begin{aligned}
\Gamma_{X} & \equiv \Gamma\left(X \rightarrow e^{+} e^{-}\right) \\
& =\frac{\alpha_{\mathrm{em}} \varepsilon_{e}^{2} m_{X}}{3}\left(1+2 r_{e}\right) \sqrt{1-4 r_{e}} \\
& =8.0 \times 10^{-8} \mathrm{MeV}
\end{aligned}
$$

with $r_{e}=m_{e}^{2} / m_{X}^{2}$. The total width quoted above corresponds to a maximum value of $\varepsilon_{e}$, discussed in the previous section. Decays of a light vector boson into neutrinoantineutrino pairs that may increase $\Gamma_{X}$ width are also allowed by kinematics and are included in some extensions of the SM involving enlarged Higgs and/or gauge sectors [7,9-13]. The relevant coupling $\varepsilon_{\nu}$ can be constrained from neutrino-electron scattering in the case of the first generation like was done in the TEXONO experiment [25] yielding to $\left|\varepsilon_{e} \varepsilon_{\nu}\right|^{1 / 2} \lesssim 7 \times 10^{-5}$ [7]. The addition of the $\nu \bar{\nu}$ channels will modify the total width of the $X$ boson by less that $0.1 \%$, and our results will remain unchanged.

The lepton pair invariant mass distribution, normalized to the radiative decay width of $H^{*} \rightarrow H \gamma$, becomes the sum of the photon and $X$-boson mediated distributions, namely [we use $\lambda(x, y, z)=x^{2}+y^{2}+z^{2}-2 x y-2 x z-2 y z$ ]:

$$
\begin{aligned}
\frac{d \Gamma\left(H^{*} \rightarrow H e^{+} e^{-}\right)}{d q^{2}}= & \frac{\alpha_{\mathrm{em}}^{2}}{72 \pi \Gamma\left(H^{*} \rightarrow H \gamma\right)} \mid G_{H^{*} H \gamma}\left(q^{2}\right) \\
& +\left.G_{H^{*} H X}\left(q^{2}\right)\right|^{2} q^{2}\left[\frac{\lambda\left(m_{H^{*}}^{2}, m_{H}^{2}, q^{2}\right)^{1 / 2}}{m_{H^{*}}}\right]^{3} .
\end{aligned}
$$

Given the very narrow width of the $X 17$ boson, the interference of the amplitudes is negligible. Indeed, the interference in the dilepton spectrum vanishes at the position of the $X 17$ and it is suppressed by more than 6 orders of magnitude relative to the one-photon contribution outside the resonance.

The lepton-pair invariant mass distributions due to photon (solid red plot) and $X 17$ boson (dashed blue curve) exchange are shown separately in Fig. 1 for the six different decay channels under consideration. The shaded bands around each curve represent the theoretical error, which are difficult to visualize in the log scale. The peak due to the production of the $X 17$ boson in each channel is not located very close to the end of the lepton-pair spectrum as it happens in the nuclear case, avoiding in this way possible end-point kinematical effects. In contradistinction to the on-shell $X 17$ production, the effect of this boson is the largest for the $D^{*+}\left(D_{s}^{*+}\right) \rightarrow D^{+}\left(D_{s}^{+}\right) e^{+} e^{-}$decay. The corresponding peaks of this boson contribution are suppressed by 1 or 2 orders of magnitude in all other cases, relative to the photon contribution. Note that we are assuming universality bounds on heavier quark $\varepsilon_{c, s, b}$ couplings; since this is a conservative assumption, the experimental study of heavy mesons transitions involving lepton pairs may serve to set bounds on these unknown couplings of the hypothetical $X 17$ boson.

Table III displays the values of the decay rates for the lepton-pair production in $H^{*} \rightarrow H$ transitions normalized to the corresponding rates of the radiative decays $H^{*} \rightarrow H \gamma$, namely

$$
R_{e e}\left(H^{*}\right) \equiv \frac{\Gamma\left(H^{*} \rightarrow H e^{+} e^{-}\right)}{\Gamma\left(H^{*} \rightarrow H \gamma\right)}
$$

where the radiative rate is given by $\Gamma\left(H^{*} \rightarrow H \gamma\right)=$ $\left(\alpha_{\mathrm{em}} / 3\right)\left|F_{H^{*} H \gamma}(0)\right|^{2}\left|\vec{p}_{\gamma}\right|^{3}$. We expect that the remaining model-dependent terms in the form factors are cancelled in this ratio (all other lepton-pair and angular distributions in the following are normalized to this radiative width). As in the case of the lepton-pair spectra, the largest contribution of the $X 17$ boson is observed for the $D^{*+}$ and $D_{s}^{*+}$ decays, making these channels the most sensitive for the observation of these light boson effects. Our calculation of the electromagnetic contribution in the case of $D_{s}^{*}$ decays yields $R_{e e}^{\gamma}\left(D_{s}^{*+}\right)=6.8 \times 10^{-3}$ is in good agreement with the experimental value $\left(7.2_{-1.6}^{+1.8}\right) \times 10^{-3}$ reported in [26] When we add the contribution of the $X 17$ boson exchange, our prediction increases to $R_{e e}^{\gamma+X}\left(D_{s}^{*}\right)=(9.8 \pm 0.6) \times 10^{-3}$, which exceeds the experimental value but it is still consistent with it within $1.4 \sigma$. Let us notice that a previous prediction of this ratio $R_{e e}\left(D_{s}^{*}\right)=6.5 \times 10^{-3}$ was estimated in Ref. [26] based on the model proposed in [27] which includes only the electromagnetic contribution.

The sensitivity of $D_{s}^{*}$ decays into lepton pairs to the effects of $X 17$ boson exchange observed in the previous paragraph suggests this channel can be useful to constrain the parameter space of the hypothetical vector boson. 

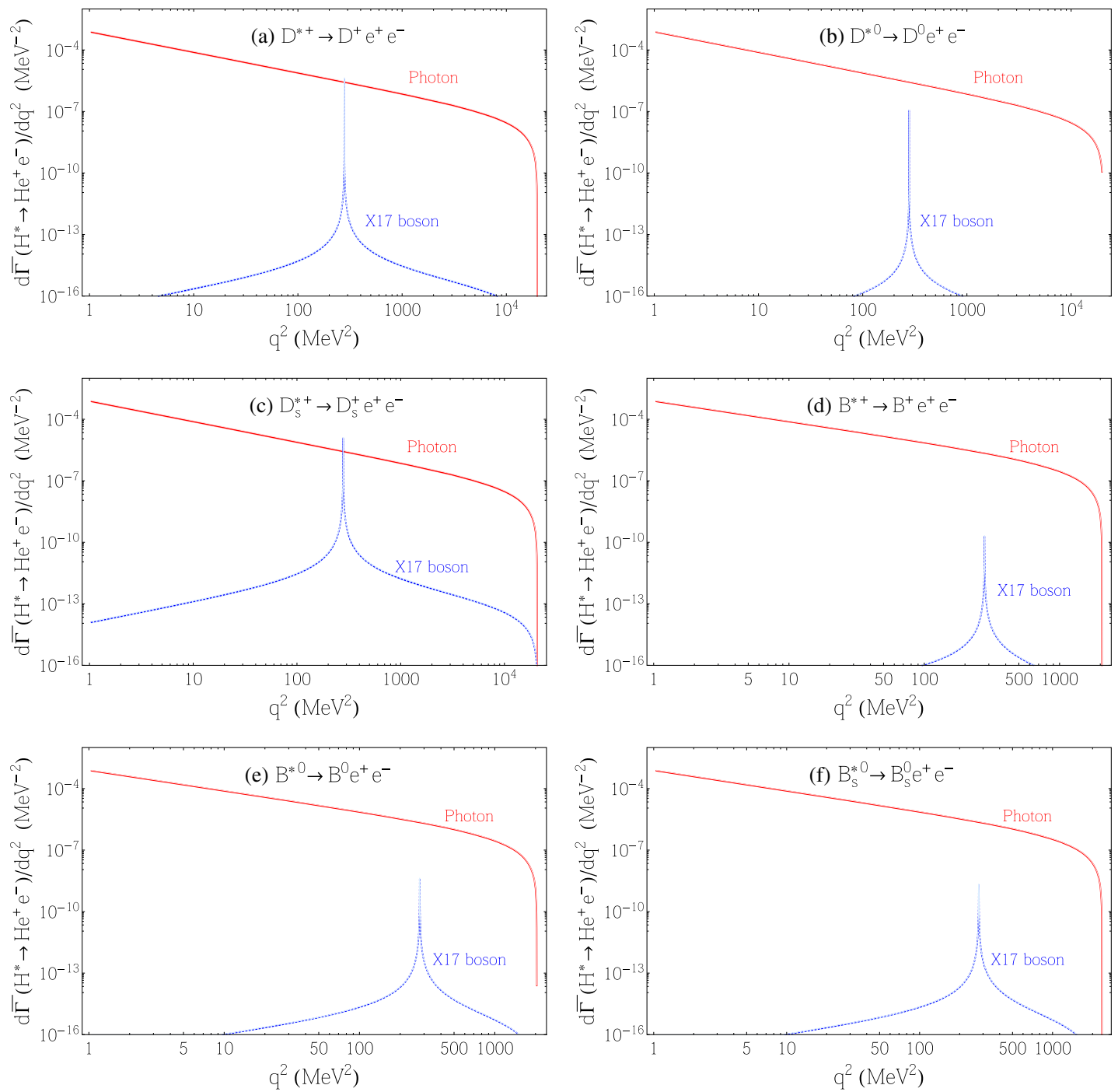

FIG. 1. Lepton pair invariant mass distributions of $H^{*} \rightarrow H e^{+} e^{-}$transitions normalized to the radiative $H^{*} \rightarrow H \gamma$ decay width: (a) $D^{*+} \rightarrow D^{+} e^{+} e^{-}$, (b) $D^{* 0} \rightarrow D^{0} e^{+} e^{-}$, (c) $D_{s}^{*+} \rightarrow D_{s}^{+} e^{+} e^{-}$, (d) $B^{*+} \rightarrow B^{+} e^{+} e^{-}$, (e) $B^{* 0} \rightarrow B^{0} e^{+} e^{-}$, and (f) $B_{s}^{* 0} \rightarrow B_{s}^{0} e^{+} e^{-}$. The red solid plot denotes the virtual photon contribution, while the $X 17$ boson contribution is represented by the blue dashed curve. The (almost invisible) shaded bands account for the theoretical uncertainties in form factors.

TABLE III. Photon and $X 17$ boson exchange contributions to the ratio of decay rates defined in Eq. (10). We assume universal couplings of the hypothetical $X 17$ boson to down-type quarks $\left[\varepsilon_{b}=\varepsilon_{s}=\varepsilon_{d}=\mp 7.4 \times 10^{-3}\right]$ and up-type quarks $\left[\varepsilon_{c}=\varepsilon_{u}= \pm 3.7 \times 10^{-3}\right]$ (see end of Sec. II). Unless explicitly indicated, theoretical uncertainties are at least 3 orders of magnitude smaller than the corresponding central values.

\begin{tabular}{|c|c|c|c|c|}
\hline Channel & $R_{e e}^{\gamma}\left(H^{*}\right)$ & $R_{e e}^{X}\left(H^{*}\right)$ & Total & Experiment \\
\hline$D^{*+} \rightarrow D^{+} e^{+} e^{-}$ & $6.67 \times 10^{-3}$ & $(1.05 \pm 0.07) \times 10^{-3}$ & $(7.72 \pm 0.07) \times 10^{-3}$ & . \\
\hline$D^{* 0} \rightarrow D^{0} e^{+} e^{-}$ & $6.67 \times 10^{-3}$ & $3.02 \times 10^{-5}$ & $6.70 \times 10^{-3}$ & 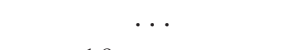 \\
\hline$D_{s}^{*+} \rightarrow D_{s}^{+} e^{+} e^{-}$ & $6.72 \times 10^{-3}$ & $(3.10 \pm 0.60) \times 10^{-3}$ & $(9.82 \pm 0.60) \times 10^{-3}$ & $\left(7.2_{-1.6}^{+1.8}\right) \times 10^{-3}[26]$ \\
\hline$B^{*+} \rightarrow B^{+} e^{+} e^{-}$ & $4.88 \times 10^{-3}$ & $(1.91 \pm 0.03) \times 10^{-5}$ & $4.90 \times 10^{-3}$ & $\ldots$ \\
\hline$B^{* 0} \rightarrow B^{0} e^{+} e^{-}$ & $4.88 \times 10^{-3}$ & $3.96 \times 10^{-4}$ & $5.28 \times 10^{-3}$ & 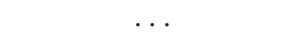 \\
\hline$B_{s}^{* 0} \rightarrow B_{s}^{0} e^{+} e^{-}$ & $4.99 \times 10^{-3}$ & $4.08 \times 10^{-4}$ & $5.40 \times 10^{-3}$ & . \\
\hline
\end{tabular}




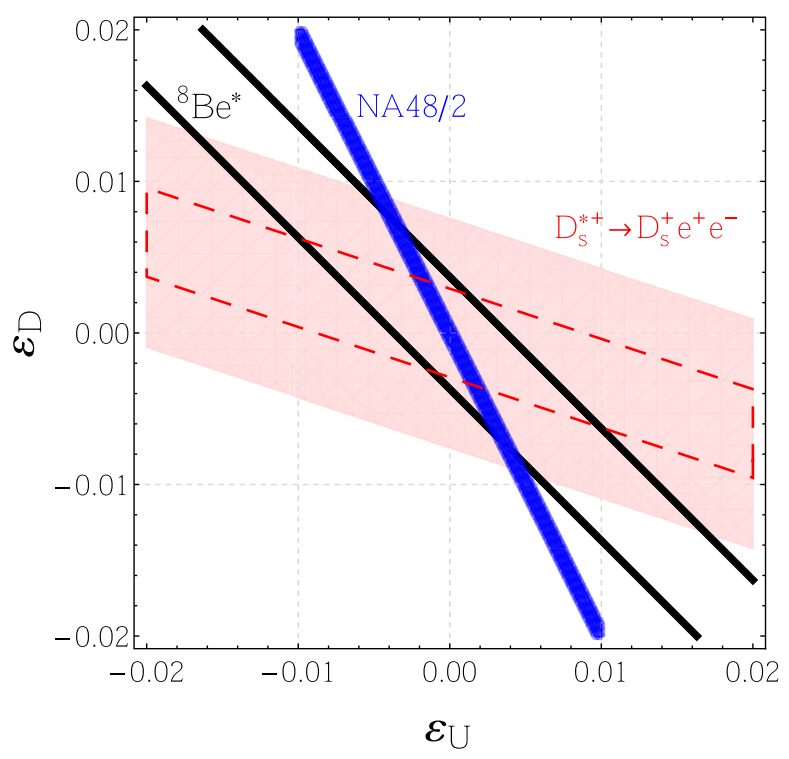

FIG. 2. The $1 \sigma$ confidence level allowed regions in the parameter space of up-type $\left(\varepsilon_{U}=\varepsilon_{c}\right)$ and down-type $\left(\varepsilon_{D}=\varepsilon_{s}\right) X$-quark couplings from $D_{s}^{*+} \rightarrow D_{s}^{+} e^{+} e^{-}$(light red shaded band). The region within dashed lines corresponds to the assumption of a fivefold improvement in the experimental uncertainty. The corresponding constraints on $\left(\varepsilon_{U}=\varepsilon_{u}, \varepsilon_{D}=\varepsilon_{d}\right)$ from the experimental results from ${ }^{8} \mathrm{Be}^{*}[5-7]$ and NA48/2 [7,23] are represented by the two parallel thin black lines and the wider steepest blue band, respectively.

In Fig. 2 we show the $1 \sigma$ confidence level allowed for the parameter space in the $\left(\varepsilon_{c}, \varepsilon_{s}\right)$ plane, obtained from the comparison of the experimental branching fraction reported by CLEO [26] and the result of integrating Eq. (9) for $D_{s}^{*} \rightarrow$ $D_{s} e^{+} e^{-}$(light red shaded band). The current experimental uncertainty in $R\left(D_{s}^{*}\right)$ is close to $25 \%$, and current experiments producing a large dataset of charmed mesons have not planned new measurements. Therefore, we will assume that a dedicated measurement of this observable may reach an improvement of the current uncertainty by a factor of 5 . Under this assumption we get the region enclosed by the red dashed contour in Fig. 2. For comparison, we also show the two thin parallel black lines corresponding to the allowed values of $\left(\varepsilon_{u}, \varepsilon_{d}\right)$ obtained from ${ }^{8} \mathrm{Be}^{*}$ results $[6,7]$ and the region allowed from the so-called "protophobic condition" obtained from the nonobservation of $\pi^{0} \rightarrow \gamma X$ by the NA48/2 experiment $[7,23]$ (single steepest blue band). The different sensitivities observed from these measurements to the up-type and down-type quark couplings makes worth an improved measurement of the heavy mesons decays discussed in this paper.
Finally, let us comment that the angular distribution of the $e^{+} e^{-}$pair, in the rest frame of the decaying particle, will be peaked closer to the collinear configuration compared to the nuclear case of ${ }^{8} \mathrm{Be}^{*}$ transitions, where $\theta\left(e^{+} e^{-}\right) \sim 140^{0}$. This happens because the $X 17$ boson is produced with a larger velocity, while in nuclear transitions this boson is produced almost at rest.

\section{CONCLUSIONS}

The hypothetical light vector boson $X 17$, proposed as a solution for the anomaly observed in lepton-pair production of ${ }^{8} \mathrm{Be}^{*}$ and ${ }^{4} \mathrm{He}$ transitions, can be studied in the clean environment provided by vector to pseudoscalar heavy mesons transitions in Belle, Belle II [28], and BESIII factories. These $H^{*}(Q \bar{q}) \rightarrow H(Q \bar{q}) e^{+} e^{-}$decays are free from theoretical uncertainties associated with nuclear effects. We have used the HQET + VMD framework to model the hadronic form factors of $1^{-} \rightarrow 0^{-}$meson transitions; however, our results are little dependent on hadronic uncertainties because the rates are normalized to the dominant $H^{*} \rightarrow H \gamma$ electromagnetic decays and the dominant contributions in most channels are dominated by photon emission off the light quarks in this model.

Although all the branching fractions of the heavy meson channels considered in this paper exhibit some sensitivity to the effects of the $X 17$ boson, decays of $D^{*+}$ and $D_{s}^{*+}$ mesons turn out to be the most sensitive ones. This happens because (i) the radiative charged charmed vector meson decay rates used as a normalization factor in $R_{e e}\left(D_{s}^{*}\right)$ and $R_{e e}\left(D^{*+}\right)$ are suppressed in the HQET + VMD owing to a partial cancellation of the heavy and light quarks contributions and, (ii) the large contribution of the light quark coupling to $X 17$ for $D^{*+} \rightarrow D^{+}$transition. Also, improved measurements of these leptonic decay channels can set additional and complementary constraints on the $X 17$ boson couplings to ordinary fermions, as shown in Fig. 2 for the case of $D_{s}^{*} \rightarrow D_{s} e^{+} e^{-}$decays or, eventually, confirm the existence of this light boson.

\section{ACKNOWLEDGMENTS}

G. L. C. acknowledges support from Ciencia de Frontera project No. 428218 (Conacyt) and PRODEP project No. 162336. The work of N.Q. has been financially supported by MINCIENCIAS and Universidad del Tolima through Convocatoria Estancias Postdoctorales No. 848-2019 (Contract No. 834-2020), and Dirección General de Investigaciones-Universidad Santiago de Cali under Project No. 935-621120-G01. 
[1] P. Fayet, U-boson production in $e^{+} e^{-}$annihilations, $\psi$ and $\Upsilon$ decays, and light dark matter, Phys. Rev. D 75, 115017 (2007).

[2] M. Pospelov, Secluded U(1) below the weak scale, Phys. Rev. D 80, 095002 (2009).

[3] R. Essig et al., Working Group Report: New light weakly coupled particles, arXiv:1311.0029.

[4] See talks at the Workshop on Feeble Interacting Particles 2020, https://indico.cern.ch/event/864648/timetable/ (2020).

[5] A.J. Krasznahorkay et al., Observation of Anomalous Internal Pair Creation in Be8: A Possible Indication of a Light, Neutral Boson, Phys. Rev. Lett. 116, 042501 (2016).

[6] J. L. Feng, B. Fornal, I. Galon, S. Gardner, J. Smolinsky, T. M. P. Tait, and P. Tanedo, Protophobic Fifth-Force Interpretation of the Observed Anomaly in ${ }^{8} \mathrm{Be}$ Nuclear Transitions, Phys. Rev. Lett. 117, 071803 (2016).

[7] J. L. Feng, B. Fornal, I. Galon, S. Gardner, J. Smolinsky, T. M. P. Tait, and P. Tanedo, Particle physics models for the $17 \mathrm{MeV}$ anomaly in beryllium nuclear decays, Phys. Rev. D 95, 035017 (2017).

[8] A. J. Krasznahorkay et al., New evidence supporting the existence of the hypothetic X17 particle, arXiv:1910.10459.

[9] L. Delle Rose, S. Khalil, and S. Moretti, Explanation of the $17 \mathrm{MeV}$ Atomki anomaly in a $\mathrm{U}(1)^{\prime}$-extended two Higgs doublet model, Phys. Rev. D 96, 115024 (2017).

[10] L. Delle Rose, S. Khalil, S. J. D. King, S. Moretti, and A. M. Thabt, Atomki anomaly in family-dependent $U(1)^{\prime}$ extension of the standard model, Phys. Rev. D 99, 055022 (2019).

[11] L. Delle Rose, S. Khalil, S. J. D. King, and S. Moretti, New physics suggested by Atomki anomaly, Front. Phys. 7, 73 (2019).

[12] O. Seto and T. Shimomura, Atomki anomaly in gauged $U(1)_{R}$ symmetric model, J. High Energy Phys. 04 (2021) 025 .

[13] T. Nomura and P. Sanyal, Explaining Atomki anomaly and muon $g-2$ in $U(1)_{X}$ extended flavour violating two Higgs doublet model, arXiv:2010.04266.

[14] A. Aleksejevs, S. Barkanova, Y. G. Kolomensky, and B. Sheff, A standard model explanation for the "ATOMKI anomaly", arXiv:2102.01127.
[15] J. L. Feng, T. M. P. Tait, and C. B. Verhaaren, Dynamical evidence for a fifth force explanation of the ATOMKI nuclear anomalies, Phys. Rev. D 102, 036016 (2020).

[16] X. Zhang and G. A. Miller, Can nuclear physics explain the anomaly observed in the internal pair production in the Beryllium-8 nucleus? Phys. Lett. B 773, 159 (2017).

[17] K. Ban, Y. Jho, Y. Kwon, S. C. Park, S. Park, and P. Y. Tseng, Search for new light vector boson using $J / \Psi$ at BESIII and Belle II, J. High Energy Phys. 04 (2021) 091.

[18] P. Ilten, J. Thaler, M. Williams, and W. Xue, Dark photons from charm mesons at LHCb, Phys. Rev. D 92, 115017 (2015).

[19] P. A. Zyla et al. (Particle Data Group), Review of particle physics, Prog. Theor. Exp. Phys. (2020), 083C01.

[20] P. Colangelo, F. De Fazio, and G. Nardulli, Radiative heavy meson transitions, Phys. Lett. B 316, 555 (1993).

[21] R. Casalbuoni, A. Deandrea, N. Di Bartolomeo, R. Gatto, F. Feruglio, and G. Nardulli, Effective Lagrangian for heavy and light mesons: Semileptonic decays, Phys. Lett. B 299, 139 (1993).

[22] B. Fornal, Is there a sign of new physics in beryllium transitions? Int. J. Mod. Phys. A 32, 1730020 (2017).

[23] J. R. Batley et al. (NA48/2 Collaboration), Search for the dark photon in $\pi^{0}$ decays, Phys. Lett. B 746, 178 (2015).

[24] X. Zhang and G. A. Miller, Can a protophobic vector boson explain the ATOMKI anomaly? Phys. Lett. B 813, 136061 (2021).

[25] M. Deniz et al. (TEXONO Collaboration), Measurement of $\bar{\nu}_{e}$-electron scattering cross section with a CsI(Tl) scintillating crystal array at the Kuo-Sheng nuclear power reactor, Phys. Rev. D 81, 072001 (2010).

[26] D. Cronin-Hennessy et al. (CLEO Collaboration), Observation of the Dalitz decay $D_{s}^{*+} \rightarrow D_{s}^{+} e^{+} e^{-}$, Phys. Rev. D 86, 072005 (2012).

[27] L. G. Landsberg, Electromagnetic decays of light mesons, Phys. Rep. 128, 301 (1985).

[28] E. Kou et al. (Belle-II Collaboration), The Belle II physics book, Prog. Theor. Exp. Phys. (2019), 123C01; Erratum, (2020), 029201. 